\title{
Personalised intervention for people with depression and severe COPD
}

George S. Alexopoulos, Dimitris N. Kiosses, Jo Anne Sirey, Dora Kanellopoulos,

Richard S. Novitch, Samiran Ghosh, Joanna K. Seirup and Patrick J. Raue

\section{Summary}

Chronic obstructive pulmonary disease (COPD) is often complicated by depression and exemplifies the challenge in managing chronic illnesses that require active patient participation in care. In a clinical trial (NCT00151372), we compared a novel personalised intervention for depression and COPD (PID-C) targeting treatment adherence with treatment as usual (TAU). In 138 patients with major depression and severe COPD, PID-C led to a higher remission rate and a greater reduction in

depressive symptoms and in dyspnoea-related disability than TAU over 28 weeks and 6 months after the last session. If replicated, PID-C may serve as a care model for patients with both depression and medical illnesses with a deteriorating course.

\section{Declaration of interest}

G.S.A. has received grant support from Forest

Pharmaceuticals; has consulted to Hoffman-LaRoche, Lilly, Pfizer and Otsuka; and has served on speakers bureaux of AstraZeneca, Avenir, Forest, Merck, Novartis and Sunovion.
Many older adults with chronic illnesses have depression, which worsens their outcomes and undermines treatment adherence. ${ }^{1}$ Chronic obstructive pulmonary disease (COPD) with co-occurring depression exemplifies the challenge in managing patients with chronic illnesses that require patient participation in care and can serve as a model for intervention development. Depression compounds the demands of COPD rehabilitation, which consists of strengthening, breathing and endurance exercises. We developed a personalised intervention for depression and COPD (PID-C), which focuses on both conditions. It draws from the 'theory of reasoned action', according to which patients weigh treatment risks and benefits ${ }^{2}$ aimed at shifting the balance in favour of treatment participation. The PID-C is administered by care managers who work with: (a) each patient to identify treatment barriers, and help them to work on their rehabilitation and take their prescribed antidepressants; and (b) the physicians in monitoring their patients' treatment and progress. This study (ClinicalTrials.gov: NCT00151372) tested the hypothesis that PID-C offered in the community is more effective than treatment as usual (TAU) in inducing remission of depression and reducing depressive symptoms and dyspnoea-related disability over 28 weeks.

\section{Method}

Participants were recruited from consecutive admissions to an acute in-patient pulmonary rehabilitation unit. They signed consent approved by the Weill-Cornell Institutional Review Board. The diagnosis of COPD was made by a pulmonologist according to American Thoracic Society Guidelines ${ }^{3}$ after examination, spirometry and other tests. Participants met criteria for unipolar major depression (SCID/DSM-IV), ${ }^{4}$ and had a score of $\geqslant 14$ on the 17-item Hamilton Rating Scale for Depression (HRSD). ${ }^{5}$ Patients with other psychiatric diagnoses (except anxiety disorders) or severe cognitive impairment (i.e. Mini-Mental State Examination $^{6}$ score $\leqslant 20$ ) were excluded. Participants were randomised into PID-C or TAU in blocks of five.

Participants in the PID-C group had their first session of PID-C $(30 \mathrm{~min})$ at discharge and the remainder in their own homes at weeks $3,4,8,12,16,20,24$, and 26 (see online supplement for a brief outline of the intervention). The PID-C care managers were social workers trained on the PID-C manual in three practice cases. The intervention targets patient-specific barriers to non-adherence in seven domains, i.e. misconceptions about COPD and depression, misunderstanding about their regimen, misattribution of depressive symptoms, hopelessness, overestimation of exercise effort, dissatisfaction with care and practical barriers. The care managers telephoned the patients' physicians and informed them of the patients' status and adherence. Physicians' recommendations for depression and COPD were not influenced by PID-C managers. For participants in the TAU group, at discharge their own physicians received a letter informing them of the diagnosis of depression.

The primary outcomes were: (a) remission of depression (HRSD $\leqslant 7$ ); (b) reduction of depressive symptoms; and (c) dyspnoea-related disability quantified with the Pulmonary Functional Status and Dyspnea Questionnaire - Modified (PFSDQ-M), with questions on degree of performance and frequency in ten activities influenced by dyspnoea. The PFSDQ-M has significant correlations with pulmonary function tests and partial pressure of oxygen $\left(\mathrm{PO}_{2}\right){ }^{7,8}$ These instruments were administered by trained raters and not the care managers. Intent-to-treat analyses were performed. Cox's proportional hazards survival analysis was used to study remission. To study depressive symptoms and dyspnoea, profiles of HRSD and PFSDQ-M from discharge to 28 and from 28 to 52 weeks were compared between PID-C and TAU using mixed-effects models. The models included time-trend parameter(s), group, and time $\times$ intervention interaction. The robustness of these models was tested by comparing them with the same models after multiple imputation, using a Markov Chain Monte Carlo approach.

\section{Results}

Pulmonary patients $(n=898)$ were screened and 138 met criteria and provided signed consent (see online Fig. DS1 for CONSORT diagram). There were no significant clinical differences among participants randomised to PID-C or TAU. During the intervention phase $12(18 \%)$ in the PID-C and $12(17 \%)$ in the TAU group died. Other attrition was $25 \%(n=17)$ in the PID-C and $17 \%(n=12)$ in the TAU group. There were no significant differences in demographics, depression and disability between those who dropped out and those who completed the study.

By the time of discharge, 88 participants had failed to remit (HRSD >7). The PID-C group had a higher remission rate (HRSD $\leqslant 7$ ) than TAU participants (Wald $\chi^{2}=5.78$, d.f. $=1$, $P=0.016$, hazard ratio $(\mathrm{HR})=2.18$; number needed to treat: 
(a)

(b)
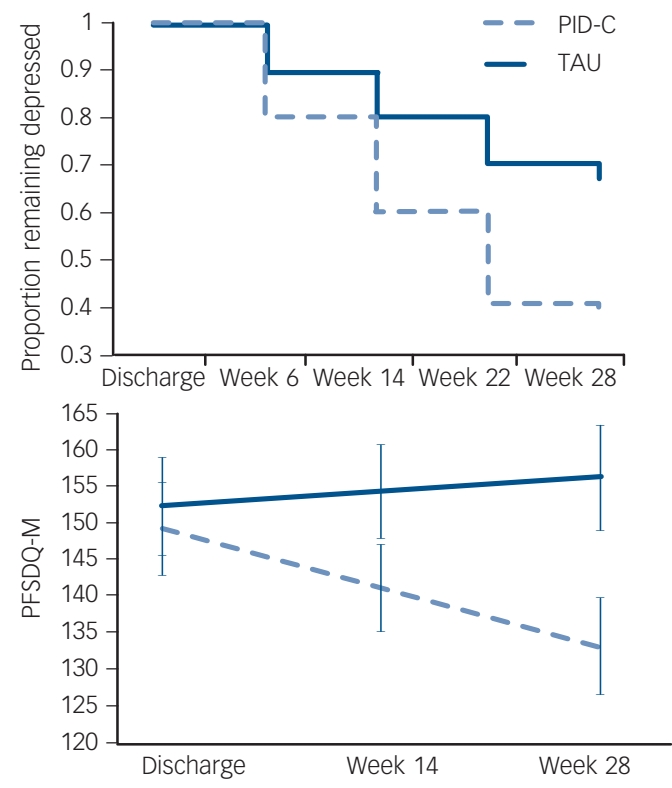

Fig. 1 (a) Remission of depression after discharge from

rehabilitation hospital and (b) course of dyspnoea-related disability in older adults with major depression and chronic obstructive

pulmonary disease (COPD) randomised to personalised intervention for depression and COPD (PID-C) or treatment as usual (TAU).

Remission of depression: 17-item Hamilton Rating Scale for Depression (HRSD) $\leqslant 7$. PFSDQ-M, Pulmonary Functional Status and Dyspnea Questionnaire - Modified.

3.83, Fig. 1). Mixed-effects modelling showed that the PID-C group had a greater decline in HRSD than those in the TAU group between discharge and 28 weeks (treatment $\times$ time: $F_{(1,396)}=5.40$; $P=0.021$ ); effect size at 28 weeks was 0.53 (95\% CI $0.09-0.97$ ). The PID-C group experienced greater reduction in dyspnoearelated disability (PFSDQ-M) than the TAU group (treatment $\times$ time: $\left.F_{(1,197)}=4.11 ; P=0.044\right)$; effect size at 28 weeks was 0.40 ( $95 \% \mathrm{CI}-0.01$ to 0.87 ) (Fig. 1).

Mixed-effects modelling showed that the PID-C group had greater decline in HRSD than the TAU group during the followup phase (treatment: $F_{(1,105)}=-2.41 ; P=0.018$ ); PID-C: least square mean at 28 weeks $9.12($ s.e. $=0.96)$ and at 52 weeks 9.44 $($ s.e. $=1.06)$; TAU: least square mean at 28 weeks 12.24 (s.e. $=0.87)$ and at 52 weeks 11.43 (s.e. $=1.02$ ). The advantage of PID-C over TAU on the PFSDQ-M was maintained even after the intervention ended (treatment $\times$ time: $t=-1.61$, d.f. $=57.5$, $P=0.113$, Fig. 1). Comparison of least square means at 52 weeks favoured PID-C $\left(t_{128}=-3.11 ; P=0.002\right)$. All comparisons cited above were similar to imputed model analyses.

\section{Discussion}

We found that PID-C led to a higher remission rate of depression ( 1 more remission every 3.83 patients), and also reduced depressive symptoms and dyspnoea-related disability more than TAU in community residing patients with major depression and severe COPD. These benefits lasted 6 months after the intervention end. Even though COPD has a deteriorating course, dyspnoearelated disability did not worsen in the PID-C group over 1 year. If replicated, PID-C may serve as a management model for the increasing numbers of people with both depression and a medical illness that requires active patient participation in care. Care management models for primary care patients with depression have been found effective ${ }^{9}$ and cost-effective. ${ }^{10}$ However, most trials targeted patients in stable medical health and have not addressed the complex needs of patients with depression who also have severe illnesses and burdensome treatment requirements. This study is unique because it targeted patients with depression at the most severe end of a deteriorating medical illness with a bleak prognosis evidenced by their high mortality $(23 \%$ in 52 weeks). As PID-C requires only brief training it could be implemented by organisations serving patients with COPD such as home healthcare and rehabilitation programmes.

Study limitations include brief assessments and high attrition, both influenced by the severity of COPD. Concerns about burden limited the number of assessments. People who are severely ill with COPD may refuse follow-up because of fatigue. Nonetheless, both arms had similar attrition. Although PID-C focused on treatment adherence, other factors, including increased physician attention, may have mediated its benefits.

In conclusion, a personalised intervention offered by care managers over a period of 28 weeks increased remission rates, and improved depressive symptoms and dyspnoea-related disability more than TAU. These benefits were sustained for an additional 6 months. A next step may be to deliver and study PID-C in a group format and facilitate its dissemination. This intervention may serve as a care model for people with both depression and a medical illness with a deteriorating course, who often neglect their own care.

George S. Alexopoulos, MD, Dimitris N. Kiosses, PhD, Jo Anne Sirey, PhD, Dora Kanellopoulos, MA, Weill Cornell Medical College Institute for Geriatric Psychiatry, White Plains; Richard S. Novitch, MD, Burke Rehabilitation Hospital, White Plains; Samiran Ghosh, PhD, Joanna K. Seirup, Patrick J. Raue, PhD, Weill Cornell Medical College Institute for Geriatric Psychiatry, White Plains, USA

Correspondence: George S. Alexopoulos, MD, Weill Cornell Medical Center, 21 Bloomingdale Road, White Plains, NY 10605, USA. Email: gsalexop@med.cornell.edu

First received 28 Aug 2012, final revision 10 Oct 2012, accepted 18 Dec 2012

\section{Funding}

NIMH R01 HLB071992, P30 MH068638, P30 MH085943 and the Sanchez Foundation. R.S.N. partially supported by a grant from the Will Rogers Institute.

\section{Acknowledgements}

We thank Timothy E. Clark and Susan Friedman, LCSW for their contributions to this study.

\section{References}

1 Lebowitz BD, Pearson JL, Schneider LS, Reynolds CF, 3rd, Alexopoulos GS, Bruce $\mathrm{ML}$, et al. Diagnosis and treatment of depression in late life. Consensus statement update. JAMA 1997; 278: 1186-90.

2 Ajzden I. The directive influence of attitudes on health behavior. In The Psychology of Action: Linking Cognition and Motivation to Behavior (ed PM Gollwitzer, JA Bargh): 385-403. Guilford Press, 1996.

3 American Thoracic Society. Standards for the diagnosis and care of patients with chronic obstructive pulmonary disease. Am J Respir Crit Care Med 1995; 152: S77-121.

4 First MB, Spitzer, Robert L, Gibbon M, Williams JBW. Structured Clinical Interview for DSM-IV-TR Axis I Disorders: Research Version, Patient Edition (SCID-I/P). Biometrics Research, New York State Psychiatric Institute, 2002.

5 Hamilton M. A rating scale for depression. I Neurol Neurosurg Psychiatry 1960; 23: 56-62.

6 Folstein MF, Folstein SE, McHugh PR. 'Mini-mental state'. A practical method for grading the cognitive state of patients for the clinician. $J$ Psychiatr Res 1975; 12: 189-98.

7 Lareau SC, Meek PM, Roos PJ. Additional testing of the modified pulmonary functional status and dyspnea questionnaire (PFSDQ-M): ease of use, stability, reliability, and validity. Am J Crit Care Med 1997; 155: A722.

8 Lareau SC, Meek PM, Roos PJ. Severe COPD: effects on activites of daily living and dyspnea. Am J Crit Care Med 1996: 153: A421.

9 Alexopoulos GS, Reynolds CF, 3rd, Bruce ML, Katz IR, Raue PJ, Mulsant BH, et al. Reducing suicidal ideation and depression in older primary care patients: 24-month outcomes of the PROSPECT study. Am J PSychiatry 2009; 166: 882-90.

10 Schoenbaum M, Unutzer J, Sherbourne C, Duan N, Rubenstein LV, Miranda J, et al. Cost-effectiveness of practice-initiated quality improvement for depression: results of a randomized controlled trial. JAMA 2001; 286: 1325-31. 